\title{
Preparation of biological samples for the recording of electrophysiological signals using high-density microelectrode arrays
}

Xinyue Yuan ( $\nabla$ xinyue.yuan@bsse.ethz.ch )

ETH Zurich

Manuel Schröter

ETH Zurich

Marie Engelene J. Obien

Michele Fiscella

Wei Gong

Tetsuhiro Kikuchi

Aoi Odawara

Shuhei Noji

Ikuro Suzuki

Jun Takahashi

Andreas Hierlemann

Urs Frey

Method Article

Keywords: Electrophysiology, high-density microelectrode arrays, extracellular recording

Posted Date: September 28th, 2020

DOI: https://doi.org/10.21203/rs.3.pex-1113/v1

License: (a) This work is licensed under a Creative Commons Attribution 4.0 International License.

Read Full License 


\section{Abstract}

The use of high-density microelectrode arrays (HD-MEAs) provides a promising approach for electrophysiological studies targeted at understanding of brain functions, profiling of neurodegenerative diseases, and drug screening. Here we present the protocol for the preparation of various biological samples for the recording of extracellular signals using HD-MEA, including primary cortical neurons, induced pluripotent stem cells (iPSCs)-derived neurons, rodent brain slices, retina, and iPSC-derived neuronal spheroids.

\section{Introduction}

\section{Reagents}

\section{Biological Samples}

Embryonic-day-18 Wistar rat

C57 Black/6 mice

\section{Chemicals}

Trypsin-EDTA (0.25\%, Life Technologies, Cat\#25200056)

Polyethylenimine (PEI, Sigma-Aldrich, Cat\#181978)

Laminin (Sigma-Aldrich, Cat\#L2020)

Poly-D-lysine (Thermo Fisher, Cat\#A3890401)

Geltrex (Thermo Fisher, Cat\#A1569601)

AMES' medium (Sigma-Aldrich, Cat\#A1420)

Artificial cerebrospinal fluid (ACSF)

4-aminopyrindine (4-AP, Sigma Aldrich, 275875)

Dimethylsulfoxide (DMSO)

Neurobasal Medium (ThermoFisher, 21103049)

DMEM/F12 (ThermoFisher, 21331-020)

Glutamax (ThermoFisher, 35050061) 


\section{Experimental Models: Cell Lines}

Human iPSC-derived glutamatergic neurons (Fujifilm Cellular Dynamics International, Cat\#01279)

Human iPSC-derived dopaminergic neurons ( Elixirgen Scientific, Cat\#DA-SeV-CW50065)

\section{Equipment}

High-density microelectrode array (HD-MEA)

Stage-top incubator (Tokai Hit)

SCAD device (Stem Cell \& Device Laboratory, Inc.)

Tissue holder (MaxWell Biosystems AG)

Vibrotome (Leica VT1200S)

\section{Procedure}

\section{Protocol for culturing primary neurons on MEAs}

1. Sterilize the MEA with $70 \%$ ethanol for $20 \mathrm{~min}$.

2. Coat the surface of MEA with $0.05 \%$ polyethylenimine (PEI, Sigma-Aldrich, USA, \#181978) and laminin (Sigma-Aldrich, \#L2020) to increase cell adhesion.

3. Prepare cell suspensions. Cells from embryonic day 18 Wistar rat cortices are dissociated in $2 \mathrm{ml}$ of trypsin with $0.25 \%$ EDTA (Invitrogen) with trituration.

4. Before plating each dish, pipet away or vacuum off most of the laminin, leaving a wet spot.

5. Drop the needed volume of cell suspension onto the laminin spot to achieve the desired cell density $\left(3000\right.$ cells $\left./ \mathrm{mm}^{2}\right)$. Leave the cells in the incubator with a cover for $30 \mathrm{~min}$ so the cells can attach to the laminin.

6. Add $1 \mathrm{~mL}$ of the plating medium at the periphery of the MEA. 
7. Maintain the cell cultures in the incubator $\left(37^{\circ} \mathrm{C}\right.$ and $5 \% \mathrm{CO}_{2} / 95 \%$ air). Exchange $50 \%$ medium every 2-3 days.

8. Cultures get mature for 2-3 weeks before experimentation, and experiments are conducted inside an incubator for a control of environmental conditions $\left(36^{\circ} \mathrm{C}\right.$ and $\left.5 \% \mathrm{CO} 2\right)$.

\section{Protocol for culturing iPSC-derived neurons on MEAs}

(following protocols provided by the supplier https://dj2jug4sd9jom.cloudfront.net/assets/CDI_iCellGlutaNeurons_AxionMEA_AP.pdf)

1. Sterilize the MEA with $70 \%$ ethanol for 20 min.

2. Coat the surface of MEA with $0.1 \mathrm{mg} / \mathrm{mL}$ poly-D-lysine and Geltrex to improve cell adhesion.

3. Thaw the iCell GlutaNeurons following protocols provided by the supplier.

4. Add the needed volume of cell suspension onto the laminin spot to achieve the desired cell density $\left(3000\right.$ cells $\left./ \mathrm{mm}^{2}\right)$. Leave the cells in the incubator with a cover for $60 \mathrm{~min}$ so the cells can attach to the laminin.

5. Slowly Add $1 \mathrm{~mL}$ of the plating medium at the periphery of the MEA.

6. Maintain the cell cultures in the incubator $\left(37^{\circ} \mathrm{C}\right.$ and $5 \% \mathrm{CO}_{2} / 95 \%$ air). Exchange $50 \%$ medium every 2 days.

7. Cultures get mature for 3-4 weeks before experimentation, and experiments are conducted inside an incubator for a control of environmental conditions $\left(36^{\circ} \mathrm{C}\right.$ and $\left.5 \% \mathrm{CO} 2\right)$.

\section{Protocol for extracellular recording from brain slices using MEAs}

1. Obtain the brain from C57 Black/6 mice (16-21 days old for cerebellar slices, 6-8 weeks old for hippocampal slices), then place in ice-cold artificial cerebrospinal fluid (ACSF) cutting solution (in [mM]: $125 \mathrm{NaCl}, 2.5 \mathrm{KCl}, 25$ glucose, $25 \mathrm{NaHCO}_{3}, 1.25 \mathrm{NaH}_{2} \mathrm{PO}_{4}, 0 \mathrm{CaCl}_{2}, 2 \mathrm{MgCl}_{2}$; the osmolarity of the solution was $\sim 310 \mathrm{mOsmol} / \mathrm{L}$, and bubbled with carboxygen: $95 \% \mathrm{O}_{2}, 5 \% \mathrm{CO}_{2}$ ).

2. Obtain coronal hippocampal slices and parasagittal cerebellum slices ( $300 \mu \mathrm{m}$ thickness) with a vibratome (Leica VT1200S).

3. The slices are then stabilized at room temperature for at least $1 \mathrm{~h}$ in the oxygenated ACSF before transferring to the MEA recording chamber for experiments. 
4. Place the acute slices on top of the array and hold in place with a membrane (Tissue Holder, MaxWell Biosystems AG, Zurich, Switzerland).

5. During the experiments, perfuse the slices with artificial cerebrospinal fluid (ACSF, in [mM]: $125 \mathrm{NaCl}$, $2.5 \mathrm{KCl}, 25$ glucose, $\left.25 \mathrm{NaHCO}_{3}, 1.25 \mathrm{NaH}_{2} \mathrm{PO}_{4}, 2 \mathrm{CaCl}_{2}, 1.9 \mathrm{MgSO}_{4}\right)$ with carbogen $\left(95 \% \mathrm{O}_{2}, 5 \% \mathrm{CO}_{2}\right)$ at $32-35^{\circ} \mathrm{C}$ with a heater (TC01, Multi-channel Systems, Reutlingen, Germany).

5. For drug application on hippocampal slices, 4-aminopyrindine (4-AP) stock solution is prepared by dissolving it in dimethylsulfoxide (DMSO) and stored at $-20^{\circ} \mathrm{C}$. 4-AP is diluted into ACSF to $100 \mu \mathrm{M}$ just before the experiments.

\section{Protocol for extracellular recording from retina using MEAs}

1. Isolate retinae from adult Wistar rats. The eyes are dissected at room temperature under dim red light conditions in Ames solution (Sigma, A1420) continuously equilibrated with $5 \% \mathrm{CO}_{2}-95 \% \mathrm{O}_{2}$.

2. Remove the vitreous, and place a retina patch with ganglion-cell-side down on the electrode array and perfuse with Ames solution $\left(\mathrm{pH} 7.4,35^{\circ} \mathrm{C}\right)$ equilibrated with $5 \% \mathrm{CO}_{2}-95 \% \mathrm{O}_{2}$.

3. Pressed the retina path onto the array with a polyester membrane (Tissue Holder, MaxWell Biosystems $A G$, Zurich, Switzerland).

4. Retinal ganglion cell extracellular activity could be recorded for $1-2$ hours.

5. For light stimulation, a LED flashlight with light pulses with a frequency of $0.5 \mathrm{~Hz}$ is used.

\section{Protocol for extracellular recording from iPSC-derived neuronal spheroids using MEAs}

1. The dopaminergic neuronal spheroids were prepared using a published protocol (DOI:

10.21203/rs.3.pex-954/v1) . The human induced pluripotent stem cells (iPSCs) were induced to form dopaminergic progenitor cells (DAPs). The steps included to start with a high-density culture, to use cell sorting whiel applying a cell surface marker for floor plate, and to establish a maturation culture to form floating aggregates.

2. Coat the surface of the array with PEI and laminin to promote tissue adhesion.

3. Conduct the experiments inside an incubator for a control of environmental conditions $\left(36^{\circ} \mathrm{C}\right.$ and $5 \%$ $\mathrm{CO}_{2}$ ). 


\section{Protocol for SCAD devices}

1. The surface of the SCAD Cell Scaffold (SCAD, SCAD-CS $\left.{ }^{T M}, C D 023\right)$ is coated with $0.002 \%$ PLO (Poly-LOrnithine, Sigma Aldrich, P4957-50ML) and 10 gg/mL laminin (Sigma Aldrich, L2020).

2. The human iPSC-derived neurons (Quick-Neuron ${ }^{\mathrm{TM}}$ Dopaminergic-Human iPSC-derived Neurons , Elixirgen Scientific, DA-SeV-CW50065) are plated onto the SCAD Cell Scaffold inside well plates at a density of 70,000 cells $/ \mathrm{cm}^{2}$.

3. The culture is plated and maintained following the protocol from the supplier (https://elixirgenscientific.com/wp-content/uploads/pdf/protocol/qndsvf.pdf). Briefly, a Medium N [Neurobasal Medium (ThermoFisher, 21103049) with DMEM/F12 (ThermoFisher, 21331-020), Glutamax (ThermoFisher, 35050061), Penicillin-Streptomycin (FUJIFILM Wako, 168-23191), and Component N] containing $10 \mu \mathrm{M}$ Y27632 (Nacalai, 08945) is used to seed cells, and the medium is changed to Medium $\mathrm{N}$ with Component D2, Component D3 on the next day and again change half volume on Day 3. Half of the medium is replaced with fresh Medium N with Component D4 and Component D5 on Day 4. Then, total amount of the medium is replaced with fresh Medium N with Component D4 and Component D6 on Day 5 and half volume on Day 7. Neurobasal Plus medium containing B-27 Plus supplement (ThermoFisher, A3653401) is used after Day 10 and thereafter. Half of the Neurobasal Plus medium is replaced every 3 or 4 day.

4. SCAD device cultured for 112 days could be mounted on HD-MEA chip and neural activities could be recorded. The SCAD device is placed and pressed onto the array with a membrane (Tissue Holder, MaxWell Biosystems AG, Zurich, Switzerland). 